\title{
Helium in first and second-generation stars in globular clusters from spectroscopy of red giants ${ }^{\star}$
}

\author{
A. Bragaglia ${ }^{1}$, E. Carretta ${ }^{1}$, R. Gratton ${ }^{2}$, V. D’Orazi ${ }^{2}$, S. Cassisi ${ }^{3}$, and S. Lucatello ${ }^{2,4,5}$ \\ 1 INAF-Osservatorio Astronomico di Bologna, Via Ranzani 1, 40127 Bologna, Italy \\ e-mail: [angela.bragaglia; eugenio.carretta]@oabo.inaf.it \\ 2 INAF-Osservatorio Astronomico di Padova, Vicolo dell'Osservatorio 5, 35122 Padova, Italy \\ e-mail: [raffaele.gratton; valentina.dorazi; sara.lucatello]@oapd.inaf.it \\ 3 INAF-Osservatorio Astronomico di Collurania, via M. Maggini, 64100 Teramo, Italy \\ e-mail: cassisi@oa-teramo.inaf.it \\ 4 Excellence Cluster Universe, Technische Universität München, Boltzmannstr. 2, 85748 Garching, Germany \\ 5 Max-Planck-Institut für Astrophysik, 85741 Garching, Germany
}

Received 1 April 2010 / Accepted 11 May 2010

\section{ABSTRACT}

\begin{abstract}
The stars in a globular cluster (GC) have always been considered coeval and of the same metallicity. Recently, this assumption has been challenged on the basis of spectroscopic and photometric observations, which show various generations of stars in GCs, differing in the abundances of products of H-burning at high temperatures. The main final product of this burning is He. It is then important to study the connections between stars properties and He content. We consider here the nearly 1400 stars on the red giant branch (RGB) observed with FLAMES@VLT in 19 Galactic Globular Clusters (GCs) in the course of our project on the Na-O anticorrelation. Stars with different $\mathrm{He}$ are expected to have different temperatures (i.e., different colours), slightly different metallicities [Fe/H], and different luminosity levels of the RGB bump. All these differences are small, but our study has the necessary precision, good statistics, and homogeneity to detect them. Besides considering the observed colours and the temperatures and metallicities determined in our survey, we computed suitable sets of stellar models - fully consistent with those present in the BaSTI archive - for various assumptions about the initial He content. We find that differences in observable quantities that can be attributed to variations in He content are generally detectable between stars of the primordial (P, first-generation) and extreme (E, second-generation) populations, but not between the primordial and intermediate ones (I). The only exception, where differences are also significant between $\mathrm{P}$ and I populations, is the cluster NGC 2808 , where three populations are clearly separated also on the main sequence and possibly on the horizontal branch. The average enhancement in the He mass fraction $Y$ between $\mathrm{P}$ and $\mathrm{E}$ stars is about $0.05-0.11$, depending on the assumptions. The differences in $Y$, for NGC 2808 alone, are about $0.11-0.14$ between P and I stars, and about $0.15-0.19$ between $\mathrm{P}$ and $\mathrm{E}$ stars, again depending on the assumptions. When we consider the RGB bump luminosity of first and second-generation stars we find different levels; the implied $Y$ difference is more difficult to quantify, but agrees with the other determinations.
\end{abstract}

Key words. stars: atmospheres - stars: Population II - globular clusters: general - stars: abundances

\section{Introduction}

Recent progress has indicated that large star-to-star variations exist in the He content among stars in globular clusters (GCs), in spite of difficulties in directly deriving $\mathrm{He}$ abundances (see however Villanova et al. 2009; Moehler et al. 2007). The first clear evidence has been obtained from observations of multiple main sequences in $\omega$ Cen (Bedin et al. 2004) and NGC 2808 (D'Antona et al. 2005; Piotto et al. 2007). In $\omega$ Cen, stars on the bluest main sequence (MS) are more metal-rich than those of the reddest one (Piotto et al. 2005), a fact that can only be explained by an He content higher by about 0.10 to 0.15 in the mass fraction $Y$ (as originally suggested by Norris 2004) with respect to a canonical (primordial) He content of about 0.245 commonly adopted for the bulk of the stellar population in this cluster. The small scatter found in NGC 2808 among red giant branch (RGB) stars also implies a larger He content for the bluer MS, by roughly the same amount. Two other clusters have

* Based on observations collected at ESO telescopes under programmes 072.D-507 and 073.D-0211. been found to have wide MSs: 47 Tuc (Anderson et al. 2009) and NGC 6752 (Milone et al. 2010, who also suggest a possible

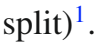

A star-to-star spread in the He abundance may explain many aspects of the horizontal branches (HBs) of GCs, as extensively discussed in Gratton et al. (2010), but we are aware that the case for a null spread of He in GCs is also maintained in the literature (e.g., Catelan 2009). Internal variations in He are likely to be connected with other chemical signatures found in GCs, related to $\mathrm{H}$ burning occurring at high temperatures. This has been first proposed in a modern way by D'Antona et al. (2002), who

\footnotetext{
1 The split subgiant branches (SGB, e.g., NGC 6388: Moretti et al. 2009; NGC 1851: Milone et al. 2008) seem more related to differences in age or CNO content. A spread in ages is probably the cause of the spread/split MS turn-offs found in many intermediate-age Magellanic Cloud GCs (Mackey et al. 2008; Milone et al. 2009, in LMC; Glatt et al. 2008, in SMC) even if for them - but notably not for the old Galactic GCs - there is an alternative physical interpretation based on the observational effects associated to the occurrence of stellar rotation as discussed by Bastian \& de Mink (2009).
} 
noticed that the lower masses of He-rich stars may naturally explain the correlation between the presence of stars at the blue extreme of the $\mathrm{HB}$, and of stars extremely depleted in $\mathrm{O}$ in various clusters. Lee et al. (2005) suggest that a super-He rich population could explain the extreme HB stars in GCs, specifically presenting the cases of $\omega$ Cen and NGC 2808 (where they had predicted a spread or a split of the MS, just before it was actually found). Kaviraj et al. (2007) suggest that the far-UV properties of GCs in the Virgo elliptical M87 could be explained by a super-He rich population similar to the one in $\omega$ Cen. D'Antona \& Caloi (2008) try to explain the whole distribution of stars along the HB of various clusters (including NGC 2808, M 13, and M 3 ) as due to star-to-star variations in the He content (but see also Castellani et al. 2005). In a companion paper (Gratton et al. 2010), we make a more systematic comparison of the extremes of the distribution of stars along the $\mathrm{HB}$ and of the $\mathrm{Na}-\mathrm{O}$ anticorrelation. We find that median HB colours are reproduced quite well by a combination of metallicity, age, and a simple mass loss proportional to metallicity. However, the low-mass extreme of the HB is clearly correlated with the extension of the $\mathrm{Na}-\mathrm{O}$ anticorrelation (see also Carretta et al. 2009a) which, in turn, seems to be linked with the cluster absolute magnitude, a proxy for cluster mass.

These correlations suggest that the multiple-generation scenario needed to explain the $\mathrm{Na}-\mathrm{O}$ anticorrelation ${ }^{2}$ is able to explain many peculiarities of GCs. As we have seen, a crucial datum in this scenario is the He content of second-generation stars. While the most spectacular evidence of a variation in the He content comes from the HB and the MS, the RGB stars are also very useful because chemical composition (but -alas- not He content) can be determined easily enough for these stars using spectroscopy, unlike faint MS stars. This allows us to cross correlate the abundances of various elements, providing significant constraints on the properties of the He producer, which have not been clearly identified yet. (Two main possible mechanisms have been proposed, intermediate/massive asymptotic giant stars - AGB - and fast-rotating massive stars - FRMS - see e.g., D’Antona \& Ventura 2007; Decressin et al. 2007, respectively.) Various properties of RGB stars are affected by the variable He abundances:

1. The temperature (at a given luminosity) of He-rich RGB stars is slightly warmer than for He-poor ones (see e.g., D'Antona et al. 2002). The difference is not large (only a few tens of K), and very accurate relative determinations are required. However, both colours and line excitation can be used to show that this really occurs.

2. The gravity (again at a given luminosity) is also different, owing to both the lower mass and higher temperature of He-rich stars. The He-rich stars have slightly lower gravity, but this effect is very small, and difficult to show, because of several complications (for instance, the greater molecular weight and reduced continuum opacity simulate a higher gravity, offsetting most of the effect expected on, e.g., ionisation equilibrium), so we will not consider it here.

3. The $[\mathrm{Fe} / \mathrm{H}]$ value is different, because of the different $\mathrm{H}$ content (broadly speaking, if $Y$ increases, $X$ decreases, hence $[\mathrm{Fe} / \mathrm{H}]$ increases; things are of course more complicated, see Sec. 2.3): this difference is small but not negligible. We

\footnotetext{
${ }^{2}$ It is generally assumed that first-generation stars have a chemical composition similar to field stars of similar age and metallicity (i.e., high $\mathrm{O}$ and $\mathrm{Mg}$, low $\mathrm{Na}$ and $\mathrm{Al}$, etc.), while second-generation stars, formed from material polluted by matter lost from the primordial population, show the abundance patterns (e.g., Na-O anticorrelation) that are unique to GCs.
}

already noticed this effect in NGC 2808 (Carretta et al. 2006, Paper I).

4. Finally, the luminosity of the RGB bump is also different: the RGB bump is more luminous for He-rich stars (Iben 1968; Salaris et al. 2006), even if also in this case the situation is more complex (see Sect. 3). We showed that possible evidence of this effect can be traced in the run of $\mathrm{Na}$ abundances along the RGB of NGC 6218 (=M 12) and NGC 6752 (Carretta et al. 2007b, Paper IV).

In this paper, we intend to look for evidence of variable He content in the sample of more than 1400 stars along the RGB of 19 GCs for which we acquired FLAMES/GIRAFFE spectra in the last few years (presented in a series of papers: see Carretta et al. 2009a,b, - Papers VII and VIII - and references therein). Several features of this data set make it very promising for the present purpose: the sample is very large; it has been analysed using a very homogenous technique; star-to-star errors in effective temperatures, usually the main source of errors in this analysis, were reduced to very low values by a carefully tailored technique. In addition, we have determined $\mathrm{Na}$ and $\mathrm{O}$ abundances for almost all these stars, allowing classification of them into three different groups according to $\mathrm{Na}$ enhancement and $\mathrm{O}$ depletion (Paper VII). We called these groups $\mathrm{P}=$ primordial, that is, stars with composition similar to that of field stars and likely belonging to the primordial population; I = intermediate, and $\mathrm{E}=$ extreme, which are stars with different degrees of $\mathrm{O}$ depletion and $\mathrm{Na}$ (likely also $\mathrm{He}$ ) enhancement. We recall here that we conservatively applied this definition only to stars where both $\mathrm{O}$ and $\mathrm{Na}$ were measured, thus reducing our sample to about 960 objects, the ones that will be used in the present paper. On the other hand, the way we analysed stars should be carefully considered when we try to extract information from our data set. The most significant assumptions we made are that stars of the same luminosity have the same effective temperature and surface gravity. Whenever needed, we explicitly consider the consequences of these assumptions, and we correct our results accordingly.

Beside observations, determining the impact of different $\mathrm{He}$ abundances requires appropriate modelling. To this purpose we used a set of BaSTI evolutionary models (Pietrinferni et al. 2004, 2006, 2009) purposely computed for this paper and employing two sets of heavy-element mixtures (see Sects. 2 and 3 for details and relations).

The structure of this paper is as follows. In Sect. 2 we present the observed differences in colour, temperature, and metallicity and derive the implied differences in He content; in Sect. 3 we compare the RGB bumps of first and second-generation stars, also deducing a different He abundance. Finally, we summarise and discuss our results in Sect. 4.

\section{Difference in Helium from RGB stars}

We may find different He values for the three populations using various indicators. To actually compute the implied $Y$ (as in the definition $X+Y+Z=1$ for the chemical composition of stellar models on mass fraction), we used relations based on the BaSTI stellar evolutionary models. We have computed an extended set of low-mass stellar models for various assumptions about the initial He content. All the stellar model predictions adopted in the present analysis have been obtained exactly in the same physical framework used for the $\mathrm{BaSTI}^{3}$ stellar model

\footnotetext{
3 The whole set of stellar models used in present work, as well as additional predictions for He-enhanced models can be retrieved from the URL site: http: //www . oa-teramo . inaf.it/BASTI
} 
A. Bragaglia et al.: He in RGB stars in globular clusters

Table 1. Difference in colour and metallicity between different populations in the 19 GCs.

\begin{tabular}{rrrrrrrrrrrrr}
\hline \hline NGC & [Fe/H] & \multicolumn{3}{c}{$(V-K)_{\mathrm{P}}$} & \multicolumn{4}{c}{$(V-K)_{\mathrm{I}}-(V-K)_{\mathrm{P}}$} & \multicolumn{2}{c}{$[\mathrm{Fe} / \mathrm{H}]_{\mathrm{P}}$} & \multicolumn{3}{c}{$[\mathrm{Fe} / \mathrm{H}]_{\mathrm{I}}$} & $\Delta[\mathrm{Fe} / \mathrm{H}]$ \\
& Pap VIII & Stars & mean & rms & stars & mean & rms & mean & rms & mean & rms & \\
\hline NGC 104 & -0.768 & 24 & 0.033 & 0.061 & 52 & -0.010 & 0.038 & -0.760 & 0.041 & -0.748 & 0.039 & 0.012 \\
NGC 288 & -1.305 & 20 & -0.004 & 0.053 & 41 & -0.013 & 0.048 & -1.230 & 0.046 & -1.223 & 0.052 & 0.007 \\
NGC 1904 & -1.579 & 17 & -0.010 & 0.050 & 16 & -0.008 & 0.063 & -1.549 & 0.042 & -1.536 & 0.042 & 0.013 \\
NGC 2808 & -1.151 & 20 & 0.001 & 0.059 & 11 & -0.046 & 0.027 & -1.167 & 0.033 & -1.097 & 0.056 & 0.070 \\
NGC 3201 & -1.512 & 31 & -0.017 & 0.063 & 54 & 0.003 & 0.063 & -1.468 & 0.061 & -1.494 & 0.053 & -0.026 \\
NGC 4590 & -2.265 & 18 & -0.014 & 0.043 & 28 & 0.002 & 0.040 & -2.215 & 0.065 & -2.236 & 0.052 & -0.021 \\
NGC 5904 & -1.340 & 21 & 0.002 & 0.046 & 53 & -0.006 & 0.043 & -1.342 & 0.042 & -1.343 & 0.034 & -0.001 \\
NGC 6121 & -1.168 & 23 & 0.179 & 0.083 & 53 & 0.008 & 0.070 & -1.310 & 0.049 & -1.302 & 0.045 & 0.008 \\
NGC 6171 & -1.033 & 10 & -0.013 & 0.058 & 16 & 0.010 & 0.087 & -1.072 & 0.053 & -1.054 & 0.072 & 0.018 \\
NGC 6218 & -1.330 & 18 & 0.000 & 0.047 & 43 & -0.024 & 0.053 & -1.302 & 0.028 & -1.302 & 0.041 & 0.000 \\
NGC 6254 & -1.575 & 31 & -0.011 & 0.058 & 48 & -0.009 & 0.064 & -1.550 & 0.072 & -1.552 & 0.057 & -0.002 \\
NGC 6388 & -0.441 & 12 & 0.007 & 0.097 & 6 & -0.035 & 0.062 & -0.427 & 0.115 & -0.389 & 0.061 & 0.038 \\
NGC 6397 & -1.988 & & & & & & & & & -2.006 & 0.067 & \\
NGC 6441 & -0.430 & 3 & -0.062 & 0.084 & 3 & 0.047 & 0.043 & -0.284 & & -0.311 & & -0.027 \\
NGC 6752 & -1.555 & 21 & -0.037 & 0.053 & 57 & 0.027 & 0.055 & -1.549 & 0.055 & -1.563 & 0.053 & -0.014 \\
NGC 6809 & -1.934 & 16 & -0.035 & 0.062 & 55 & 0.017 & 0.053 & -1.946 & 0.042 & -1.955 & 0.057 & -0.009 \\
NGC 6838 & -0.832 & 11 & -0.001 & 0.042 & 19 & -0.005 & 0.041 & -0.803 & 0.044 & -0.808 & 0.038 & -0.005 \\
NGC 7078 & -2.320 & 12 & -0.029 & 0.032 & 18 & 0.003 & 0.069 & -2.312 & 0.066 & -2.320 & 0.050 & -0.008 \\
NGC 7099 & -2.344 & 11 & -0.039 & 0.048 & 13 & 0.023 & 0.059 & -2.313 & 0.046 & -2.340 & 0.053 & -0.027 \\
\hline
\end{tabular}

library (Pietrinferni et al. 2004, 2006). Thus, they are fully consistent with the predictions corresponding to a "canonical" assumption about the initial He abundance $(0.245 \leq Y \leq 0.27)$. In particular, we used two assumptions for the heavy element mixtures (others may be valid, but these represent two extremes):

(a) one where the second-generation I, E stars have the same heavy-element distribution of the first-generation P stars, both $\alpha$-enhanced (for more details see Pietrinferni et al. 2006) and without peculiarities in the distribution of $\mathrm{C}, \mathrm{N}$, $\mathrm{O}$, and $\mathrm{Na}$ (a simplified approach);

(b) a second one where the I, E stars have a peculiar distribution of metals (i.e., showing a signature of the Na-O anticorrelation) and different from the standard one of the $\mathrm{P}$ stars. In particular, we adopted the "CNONa-extreme" chemical composition accounted for by Pietrinferni et al. (2009), which has the $\mathrm{C}+\mathrm{N}+\mathrm{O}$ sum enhanced by a factor of two.

It is worth mentioning that, for a fixed iron content $[\mathrm{Fe} / \mathrm{H}]$, the global metallicity $Z$ is higher for case $b$ than for case $a$ mainly because the sum of $(\mathrm{C}+\mathrm{N}+\mathrm{O})$ is enhanced with respect the "normal" $\alpha$-enhanced mixture. This has some implications: for the same $\mathrm{He}$, the temperature of a CNONa-extreme track is lower than the corresponding normal $\alpha$-enhanced and its RGB bump is fainter (see Sect. 3). This also means that we cannot give a simple and straightforward interpretation of differences e.g., in colour or RGB bump brightness as differences in He because the result also depends on the mixture of heavy elements we attribute to the second-generation stars.

\section{1. $\Delta Y$ from difference in colours}

For all stars in our 19 GCs we have Johnson $V$ magnitudes. The photometry was obtained as described in Papers I to VIII of our series $^{4}$ : Carretta et al. (2006, 2007a,b,c, 2009a,b) and Gratton et al. $(2006,2007)$, where the interested reader can find references to the original papers or description for the unpublished

\footnotetext{
${ }^{4}$ In particular: in Paper I for NGC 2808, Paper II for NGC 6752, Papers III, V for NGC 6441, Paper IV for NGC 6218, Paper VI for NGC 6838, Paper VII for NGC 104, NGC 288, NGC 1904, NGC 3201, NGC 4590, NGC 5904, NGC 6121, NGC 6171, NGC 6254, NGC 6397, NGC 6809, NGC 6838, NGC 7078, and NGC 7099.
}

data. We retrieved $K$ magnitudes from the $2 \mathrm{MASS}^{5}$ Point Source Catalog (Skrutskie et al. 2006) for almost all stars. Distance moduli and colour excesses $E(B-V)$ were taken from the on-line version of the GC catalogue by Harris (1996).

In our series of papers devoted to the study of $\mathrm{Na}$ and $\mathrm{O}$ abundances for RGB stars, we derived the temperatures of the stars using the Alonso et al. (1999) relations and the photometry. In particular, to decrease the internal error, we derived the temperatures not directly from the $(V-K)$ colours, but from relations of temperature with the $V$ or $K$ magnitude (see Papers II to VIII; the exception is NGC 2808, the first cluster analysed, for which we used the classical relation between colour and temperature). In the present paper we used the unreddened $(V-K)$ colours. The second step provided the necessary elimination of offsets between the photometries: in each cluster we selected stars belonging to the $\mathrm{P}$ component, computed the average offset in colour between $(V-K)$ observed and derived from the colourtemperature relations, and used it to define the zero point for the $\mathrm{P}$ stars (so that they have zero colour by definition).

Only after this normalisation did we compute the offsets in colours between the three populations. We have 319, 587, and 39 stars in the P, I, E components, respectively. We only considered stars fainter than $M_{K}=-3.5$ because for brighter stars the different sequences for different $\mathrm{He}$ are too close and become indistinguishable ${ }^{6}$.

Results for the 19 GCs are given in Table 1, where we indicate the cluster, the metallicity (Col. 2, taken from Paper VIII), the number of stars in the $\mathrm{P}$ component (Col. 3), their average colour $(V-K)_{\mathrm{P}}$ and rms (Cols. 4,5$)$, the number of stars in the I component (Col. 6), and their average offset with respect to the

\footnotetext{
5 The Two Micron All Sky Survey is a joint project of the University of Massachusetts and the Infrared Processing and Analysis Center/California Institute of Technology, funded by the National Aeronautics and Space Administration and the National Science Foundation.

6 This has also the effect of minimising the possible contamination of AGB stars, since the separation between RGB and AGB is clear at that magnitude level. As an example, in the case of NGC 6752, $M_{K}=$ -3.5 means $V \sim 12.4$ (see Carretta et al. 2007a). From Fig. 1 of that paper, the separation in colour $(B-V)$ between the two sequences is then about $0.1 \mathrm{mag}$, i.e., much more than the photometric error.
} 


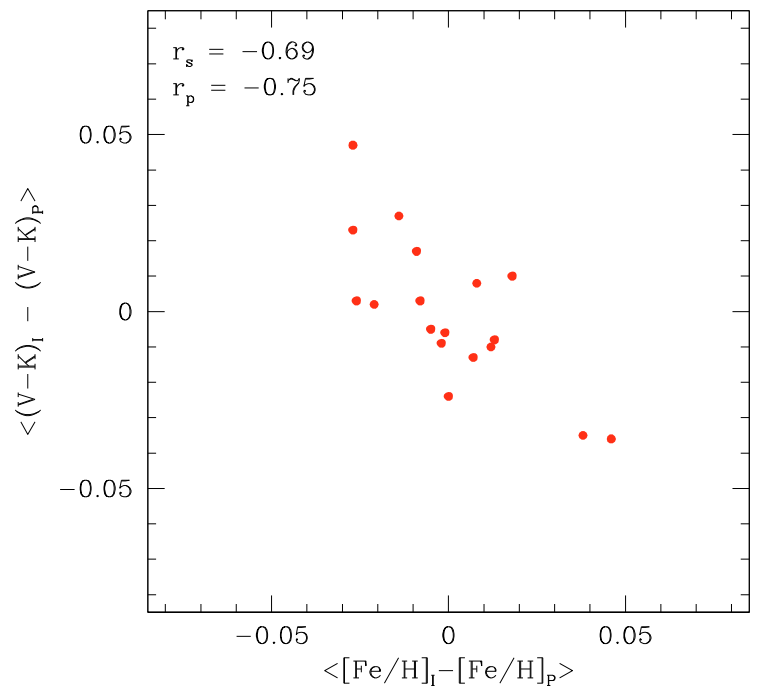

Fig. 1. Mean differences in $(V-K)$ colours between the I and $\mathrm{P}$ components in the $19 \mathrm{GCs}$ as a function of the metallicity difference (I-P). The two are well-correlated, as indicated by the Pearson and Spearman coefficients.

P stars, with rms (Cols. 7, 8). Columns 9 to 12 show the average metallicities of the $\mathrm{P}$ and I components with their rms, and Col. 13 shows the difference in $[\mathrm{Fe} / \mathrm{H}]$ (see next section). We did not evaluate the corresponding individual cluster values for the E component since the number of E stars is very small, and they would not be significant. Figure 1 shows that differences in colours and in metallicity are anticorrelated well as indicated by the Pearson and Spearman rank coefficients, and this is significant at better than the $99.9 \%$ level.

To see whether there is a difference in colour among the different populations, we summed up the $\mathrm{P}, \mathrm{I}$, and $\mathrm{E}$ components in all clusters and obtained the following weighted means ${ }^{7}$ :

$$
(V-K)_{\mathrm{I}}-(V-K)_{\mathrm{P}}=-0.003 \pm 0.004(\mathrm{rms}=0.060)
$$$$
(V-K)_{\mathrm{E}}-(V-K)_{\mathrm{P}}=-0.037 \pm 0.010(\mathrm{rms}=0.056) \text {. }
$$

We see that $\Delta(V-K)$ is nearly the same as the error, hence not significant, for the $\mathrm{P}$ and I stars, while it is about 4 times the error for the $\mathrm{P}$ and $\mathrm{E}$ stars. We conclude that the extreme populations is significantly bluer than the primordial one. This $\Delta(V-K)$ corresponds to a $\Delta Y$ of about 0.05 or 0.08 , according to the models cited, for cases $a$ ) and $b$ ), respectively. The differences in colour and in metallicity (see Sect. 2.3) are summarised in Table 2, while the corresponding $\Delta Y$ values are shown in Table 3, for simplicity.

A complication may arise from the presence of $\mathrm{CO}$ bands in the $K$ filter. They are stronger in $\mathrm{P}$ stars than in O-depleted $\mathrm{I}$ and $\mathrm{E}$ ones, and may depress the flux in the $K$ filter by about $1-2 \%$, making the P stars to appear bluer, hence decreasing the difference in $(V-K)$ among populations. From simulations based on the CO index by Cohen et al. (1978) we have estimated that the $(V-K)$ of $\mathrm{P}$ stars should be about 0.013 mag redder than they appear. That means that the corrected $\Delta(V-K)$ between $\mathrm{E}$ and $\mathrm{P}$ is about -0.05 ; this corresponds to $\Delta Y$ of about 0.06 or 0.10 , for the two mixtures.

\footnotetext{
7 The values would become $-0.003 \pm 0.004$ and $-0.042 \pm 0.008$ had we eliminated NGC 3201, a cluster with differential reddening (which we corrected for anyway, using the maps by von Braun \& Mateo 2001). Its potential influence on the global properties of our sample is greater than the one by, e.g., NGC 6388 or NGC 6441 because we have many more stars. Even so, the results are indistinguishable.
}

Table 2. Summary of differences in colour and metallicity between populations.

\begin{tabular}{lcccc}
\hline \hline Sample & $\Delta(V-K)_{\mathrm{IP}}$ & $\Delta(V-K)_{\mathrm{EP}}$ & $\Delta[\mathrm{Fe} / \mathrm{H}]_{\mathrm{IP}}$ & $\Delta[\mathrm{Fe} / \mathrm{H}]_{\mathrm{EP}}$ \\
\hline all & -0.003 & -0.037 & 0.000 & 0.027 \\
${\text { all }(+ \text { corr })^{1}}_{\text {NGC 2808 }}$ & - & -0.050 & - & - \\
NGC 2808(+corr $)^{1}$ & -0.036 & -0.044 & 0.046 & 0.084 \\
\hline
\end{tabular}

Notes. ${ }^{1}$ With correction for the CO bands.

This is a global result, averaged over the $19 \mathrm{GCs}$; it is interesting to consider NGC 2808 separately, the only cluster in our sample for which large differences in He have been deduced on the basis of the multiple MSs (Piotto et al. 2007). We find for NGC 2808

$$
\begin{aligned}
& (V-K)_{\mathrm{I}}-(V-K)_{\mathrm{P}}=-0.036 \pm 0.015 \\
& (V-K)_{\mathrm{E}}-(V-K)_{\mathrm{P}}=-0.044 \pm 0.017
\end{aligned}
$$

For this cluster both the $\mathrm{I}-\mathrm{P}$ difference and the $\mathrm{E}-\mathrm{P}$ one are significant at about the $2.5 \sigma$ level. These $\Delta(V-K)$ imply a $\Delta Y$ of about 0.05 (case $a$ ) or 0.08 (case $b$ ), and 0.08 (0.09) between the $\mathrm{P}$ and I or E populations, respectively. Taking again the effect of $\mathrm{CO}$ bands into account, these differences become $\Delta(V-K)_{\mathrm{I}-\mathrm{P}}=$ -0.049 and $\Delta(V-K)_{\mathrm{E}-\mathrm{P}}=-0.057$ which translate into $\Delta Y_{\mathrm{I}-\mathrm{P}}=$ $0.06(0.10)$ and $\Delta Y_{\mathrm{E}-\mathrm{P}}=0.07(0.11)$, respectively ${ }^{8}$.

\section{2. $\Delta Y$ from difference in $d \theta$}

As an alternative to the use of temperatures derived from colours, we may also use information derived from excitation to find (small) temperature differences between the three populations 9 . This is possible because we did not derive $T_{\text {eff }}$ 's from the excitation equilibrium (i.e. the spectroscopic route) but from the photometry. From the excitation equilibrium we derived the $\mathrm{d} \theta$ values for all the programme stars; recalling that $\mathrm{d} \theta>0$ means that the star is warmer than expected from its luminosity, we found the following weighted average values: $d \theta_{\mathrm{E}}-\mathrm{d} \theta_{\mathrm{P}}=$ $+0.0092 \pm 0.0040$ using the $39 \mathrm{E}$ stars and the $207 \mathrm{P}$ stars with more than 25 lines measured. The result improves to $\mathrm{d} \theta_{\mathrm{E}}-\mathrm{d} \theta_{\mathrm{P}}=$ $+0.0094 \pm 0.0037$ if we relax the request on the number of lines and use the $61 \mathrm{E}$ stars and the $315 \mathrm{P}$ stars with more than 15 lines measured. This difference is significant at $2.5 \sigma$ and corresponds to a difference in temperature of $32 \pm 14 \mathrm{~K}$ (in the sense of $\mathrm{E}$ stars being warmer than $\mathrm{P}$ ones) at $4500 \mathrm{~K}$, the mean temperature of the stars considered. This implies a $\Delta Y$ of about 0.06 between $\mathrm{E}$ and $\mathrm{P}$ stars (an average between results for the cases $a$ ) and $b$ ) discussed above). Since in this case we have averaged stars of all magnitudes and the sequences tend to converge at brighter luminosity, the average difference is smaller than the one implied by colours.

Once again, the difference between the $\mathrm{P}$ and I populations is not significant; the corresponding differences are $\mathrm{d} \theta_{\mathrm{I}}-\mathrm{d} \theta_{\mathrm{P}}=$ $-0.0037 \pm 0.0017$ and $\mathrm{d} \theta_{\mathrm{I}}-\mathrm{d} \theta_{\mathrm{P}}=-0.0018 \pm 0.0018$ considering stars with at least 25 or 15 lines measured, respectively.

\footnotetext{
8 The small difference in $\Delta Y_{\mathrm{E}-\mathrm{P}}$ between the average of $19 \mathrm{GCs}$ and NGC 2808 alone comes from the latter making a strong contribution to the global E population.

9 The definition is $\theta_{\mathrm{exc}}=5040 / T_{\mathrm{exc}}$. Since $T_{\mathrm{exc}} \simeq 0.86 \times T_{\mathrm{eff}}$, we have $\theta_{\mathrm{exc}}=5860 / T_{\mathrm{eff}}$. By differentiating the equation, we have the relation between $\mathrm{d} \theta$ and $\mathrm{d} T_{\text {eff. }}$.
} 
Table 3. Summary of differences in $Y$ between first and second-generation stars.

\begin{tabular}{|c|c|c|c|c|c|c|c|c|}
\hline Sample & $\Delta Y_{\mathrm{PI}}(V-K)$ & $\Delta Y_{\mathrm{PE}}(V-K)$ & $\Delta Y_{\mathrm{PI}}[\mathrm{Fe} / \mathrm{H}]$ & $\Delta Y_{\mathrm{PE}}[\mathrm{Fe} / \mathrm{H}]$ & $\Delta Y_{\mathrm{PI}}(V-K)$ & $\Delta Y_{\mathrm{PE}}(V-K)$ & $\Delta Y_{\mathrm{PI}}[\mathrm{Fe} / \mathrm{H}]$ & 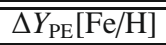 \\
\hline & \multicolumn{4}{|c|}{ Case $a$} & \multicolumn{4}{|c|}{ Case $b$} \\
\hline all & $\ldots$ & 0.049 & $\ldots$ & 0.046 & $\ldots$ & 0.077 & $\ldots$ & 0.11 \\
\hline all $(+ \text { corr. })^{1}$ & $\ldots$ & 0.064 & ... & & $\ldots$ & 0.100 & ; & $\ldots$ \\
\hline NGC 2808 & 0.047 & 0.057 & 0.109 & 0.146 & 0.075 & 0.089 & 0.14 & 0.19 \\
\hline NGC 2808(+corr. $)^{1}$ & 0.063 & 0.072 & $\ldots$ & $\ldots$ & 0.098 & 0.113 & $\ldots$ & $\ldots$ \\
\hline
\end{tabular}

Notes. ${ }^{1}$ With correction for the $\mathrm{CO}$ bands.

\section{3. $\Delta Y$ from difference in $[\mathrm{Fe} / \mathrm{H}]$}

Grossly speaking, given the definition $X+Y+Z=1$, for a fixed global metallicity $Z$, an increase in $\mathrm{He}(Y)$ has to be associated to a decrease of the $\mathrm{H}$ abundance, so that $[\mathrm{Fe} / \mathrm{H}]$ increases. Of course, the issue is more complex, since a change in the $\mathrm{H}$ to $\mathrm{He}$ ratio has effects on the star structure, which are taken into account by the evolutionary models used in the present paper.

We noticed this feature in NGC 2808 (Carretta et al. 2006) when, dividing stars in a way different from the one adopted here, we found slightly different (and increasing) values of $[\mathrm{Fe} / \mathrm{H}]$ for "O-normal", intermediate, and very O-poor stars (i.e., with canonical, intermediate, and enhanced $\mathrm{He}$ in the usual interpretation of $\mathrm{Na}-\mathrm{O}$ anti-correlation). We explore here the whole sample of our programme clusters.

In this case we have to proceed in a slightly different way. As mentioned in Sect. 2.1, in our series of papers (Papers II to VII, but notably not in Paper I on NGC 2808), we derived the temperatures used to obtain $[\mathrm{Fe} / \mathrm{H}]$ and other abundance ratios from a calibration between magnitude and colour-based temperature. This reduced the effect of errors (since magnitudes are more reliably measured than colours) and allowed to have smaller internal errors, but also effectively collapsed all possible differences in metallicity due to different He, which would have shown up as different colours, hence temperatures. Before comparing $[\mathrm{Fe} / \mathrm{H}]$ values of the different populations, we then applied a correction to transform the metallicities to the values they would have had, had we used the colour-based $T_{\text {eff }}$ 's directly.

When we apply the same procedure adopted for colours, i.e. normalising to the $\mathrm{P}$ population of each cluster (for a total of 320 stars), we obtain the following weighted means:

$[\mathrm{Fe} / \mathrm{H}]_{\mathrm{I}}-[\mathrm{Fe} / \mathrm{H}]_{\mathrm{P}}=0.000 \pm 0.003(\mathrm{rms}=0.051)$

$[\mathrm{Fe} / \mathrm{H}]_{\mathrm{E}}-[\mathrm{Fe} / \mathrm{H}]_{\mathrm{P}}=0.027 \pm 0.010(\mathrm{rms}=0.059)$

with 587 stars in the I and 38 stars in the E component, respectively. If we eliminate NGC 3201 because of its differential reddening, things do not change much: these two numbers become $0.002 \pm 0.004(288$ stars, $\mathrm{rms}=0.055)$ and $0.032 \pm 0.010(32$ stars, rms $=0.059)$, respectively. Only the difference between $\mathrm{E}$ and $\mathrm{P}$ populations is significant and, for the two cases, it implies a $\Delta Y \simeq 0.05$ (or 0.06, without NGC 3201) if the whole difference is due to the denominator in $[\mathrm{Fe} / \mathrm{H}]$, i.e., if the distribution of heavy elements is the same in $\mathrm{P}$ and $\mathrm{E}$ stars (case $a$ ). The situation is more complicated in case $b$, where $Z$ also changes. We ran models with different assumptions for the change in $Z$ (from zero to 0.002 ), and a $\Delta[\mathrm{Fe} / \mathrm{H}]=0.03$ implies for an average difference in $Z$ of 0.0013 , a $\Delta Y \simeq 0.11$.

Also in this case we may separate NGC 2808, and we obtain

$[\mathrm{Fe} / \mathrm{H}]_{\mathrm{I}}-[\mathrm{Fe} / \mathrm{H}]_{\mathrm{P}}=0.046 \pm 0.020(\mathrm{rms}=0.066)$

$[\mathrm{Fe} / \mathrm{H}]_{\mathrm{E}}-[\mathrm{Fe} / \mathrm{H}]_{\mathrm{P}}=0.084 \pm 0.018(\mathrm{rms}=0.052)$.

Both values are significant and imply $\Delta Y_{\mathrm{I}-\mathrm{P}}=0.11$ and $\Delta Y_{\mathrm{E}-\mathrm{P}}=0.15$ (case $a$ ). For case $b$, these values become, again

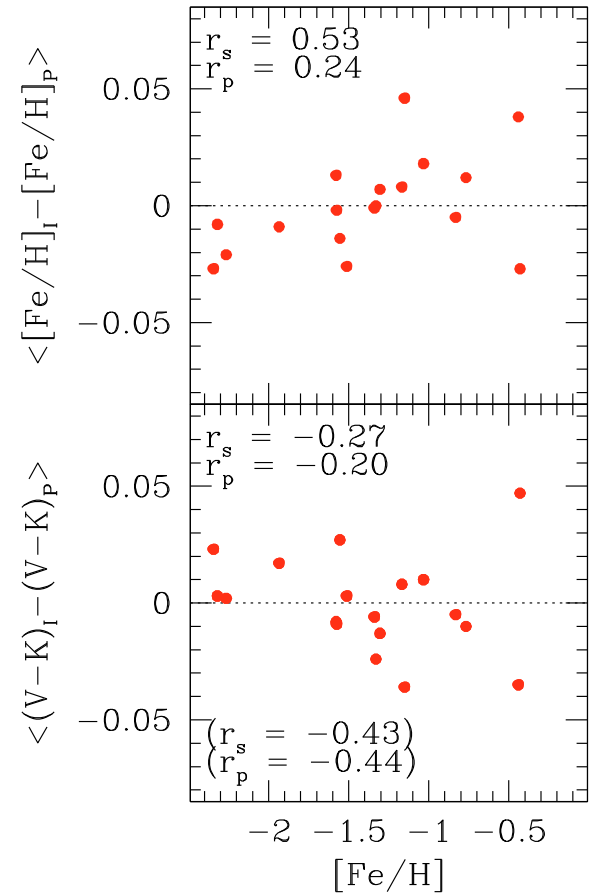

Fig. 2. Individual differences $\mathrm{I}-\mathrm{P}$ of metallicity (upper panel) and colours (lower panel) versus [Fe/H], with Spearman and Pearson coefficients indicated (in the lower panel, values in parenthesis are obtained excluding NGC 6388 and NGC 6441).

assuming the same difference in $Z$ as above, $\Delta Y_{\mathrm{I}-\mathrm{P}}=0.14$ and $\Delta Y_{\mathrm{E}-\mathrm{P}}=0.19$

\subsection{Differences among the three populations}

Even if this is part of our global study of the anticorrelations in GCs, we have to remember that $\Delta Y$ is not a direct synonym of anticorrelation and that the same extension of the anticorrelations (as measured, e.g. from the interquartile range IQR[O/Na], see Carretta et al. 2006, 2007d), may be reached for different values of $\Delta Y$. We have an indication of that from Fig. 2: in the lower panel we plot the difference in metallicity and colour between I and P stars as a function of cluster metallicity, while in the upper panel we do the same for differences in metallicity. The individual values have large errors (so we considered only the averages over the whole sample); however, there is a hint that the effects of differences in He content (as inferred from the difference in metallicity or colour) are less evident for the metal-poor clusters. Only in the metal-richer clusters are the I stars bluer and metal-richer than the $\mathrm{P}$ ones. This could suggest that, for the same production of proton-capture elements (e.g., destruction of $\mathrm{O}$ and production of $\mathrm{Na}$ ), more $\mathrm{He}$ is produced in metal-richer clusters. In other words, in the metal-poor GCs a smaller $\Delta Y$ is required to produce an effect on the shape of the 
A\&A 519, A60 (2010)
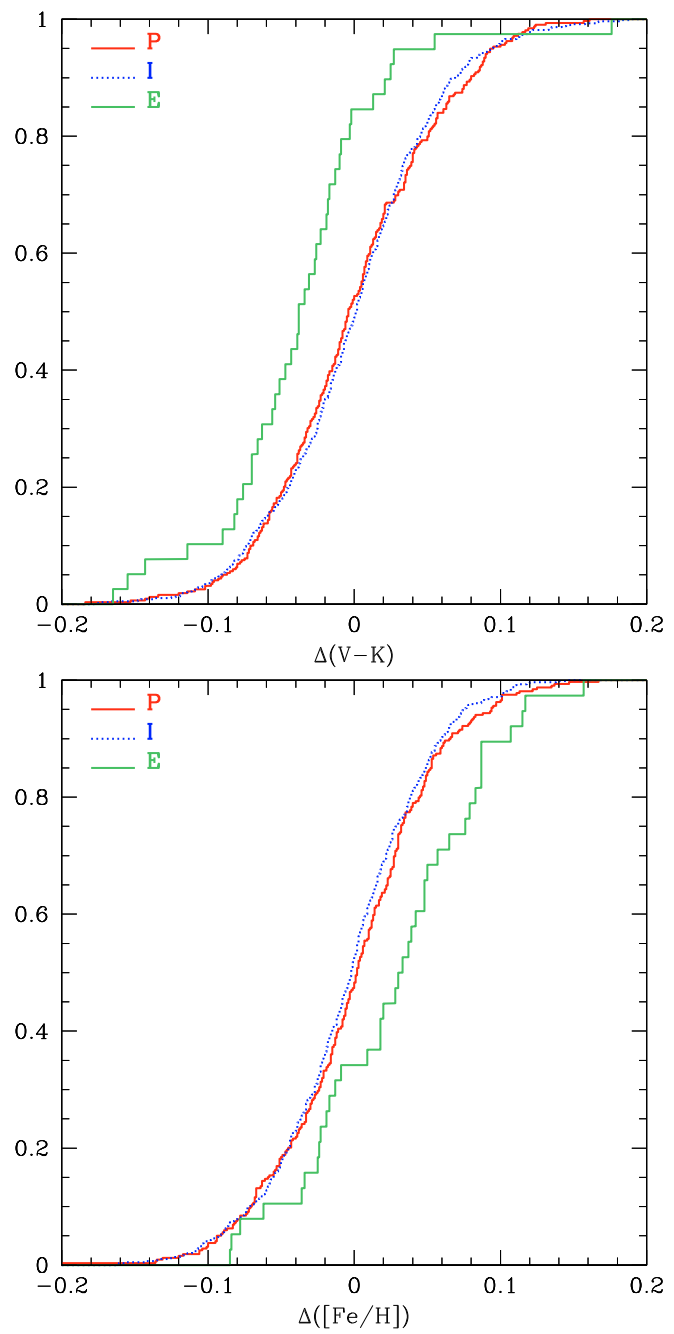

Fig. 3. Cumulative distributions for difference in colour (upper panel) and metallicity (lower panel) for individual stars in the three populations.

$\mathrm{Na}-\mathrm{O}$ anticorrelation, i.e., on the classification of a star in the I or even E population.

While the effect discussed above needs corroboration, a solid conclusion is that, with the exception of NGC 2808, the differences in colour and metallicity are only significant between the extremes of the GC populations. This can be immediately visualised using the cumulative distributions of the differences in colour and metallicity for the individual stars (i.e., the difference between each star and the average of the $\mathrm{P}$ population). Figure 3 shows these cumulative distributions, separated for $\mathrm{P}, \mathrm{I}$, and $\mathrm{E}$ stars. The P and I distributions look very similar in both panels, while the E stars show a distinct behaviour. (The probability that they are taken from the same parent distribution of the $\mathrm{P}$ stars is very low: $4 \times 10^{-4}$ and 0.015 for colour and metallicity, respectively.) As a whole, they are bluer and metal-richer.

\section{Helium from the luminosity function of Na-poor and Na-rich stars}

With the intent of finding additional evidence supporting our scenario for the correlation between Na-rich stars and their initial helium content, we also tested the possibility of detecting any brightness difference between the RGB bumps corresponding to the two distinct stellar populations. In fact, canonical
Table 4. Adopted values of $V_{\text {bump }}$.

\begin{tabular}{cccc}
\hline \hline GC & $V_{\text {bump }}$ & \pm & Reference \\
\hline NGC 0288 & 15.45 & 0.05 & 1 \\
NGC 1904 & 16.00 & 0.04 & 2 \\
NGC 3201 & 14.55 & 0.05 & 1 \\
NGC 4590 & 15.15 & 0.05 & 1 \\
NGC 5904 & 15.00 & 0.05 & 1 \\
NGC 6121 & 13.40 & 0.10 & 1 \\
NGC 6171 & 15.85 & 0.05 & 1 \\
NGC 6218 & 14.60 & 0.07 & 1 \\
NGC 6254 & 14.65 & 0.05 & 1 \\
NGC 6397 & 12.60 & 0.10 & 3 \\
NGC 6752 & 13.65 & 0.05 & 1 \\
NGC 6809 & 14.15 & 0.05 & 1 \\
NGC 6838 & 14.80 & 0.15 & 1 \\
NGC 7078 & 15.25 & 0.05 & 1 \\
\hline
\end{tabular}

References. (1) Ferraro et al. (1999); (2) Zoccali et al. (1999); (3) Alves \& Sarajedini (1999).

stellar evolution predicts that the RGB bump brightness increases when the initial He content increases (see, for instance Salaris \& Cassisi 2005). As a consequence, one should expect to observe this occurrence when comparing the primordial stellar component with the intermediate/extreme one (e.g., Salaris et al. 2006).

However, the possibility of detecting this observational signature in the luminosity function (LF) of a given GC is generally strongly hampered by the inadequate statistics. (The number of RGB stars in the various LF bins is not large enough.) We made a first explorative attempt (Paper IV), combining the samples in NGC 6218 and NGC 6752 to improve the statistics. Here, in order to better overcome this problem, we decided to combine the data for the LFs corresponding to the various GCs in our database. We took the values for $V_{\text {bump }}$ from the literature (see Table 4) but could only apply the test to 14 of the 19 GCs. We did not reach the level of the bump in some of our target clusters, either because they were very far, like NGC 6388 and NGC 6441, or because they were so rich, like NGC 104 and NGC 2808, that we had enough target in the upper RGB and did not need to reach down along the RGB. (Our original goal was to obtain good spectra to measure $\mathrm{Na}$ and $\mathrm{O}$ abundances for about 100 stars per cluster, so we selected preferentially bright targets.) Finally, we did not find a $V_{\text {bump }}$ value for NGC 7099. Since it has the same metallicity as NGC 7078, we could have adopted the value for the latter, taking the distance moduli into account, but we would have introduced an additional uncertainty, given also the slight difference in age (Carretta et al. 2009c; these two GCs have the same age in Marín-Franch et al. 2009).

We have in total 1368 stars in the 14 GCs with a measured $\mathrm{Na}$ abundance. To separate $\mathrm{P}$ stars from I and $\mathrm{E}$ ones we used $[\mathrm{Na} / \mathrm{Fe}]_{\min }+0.3$ (see Carretta et al. 2009a); we have $438 \mathrm{P}$ stars and $930 \mathrm{I}$, E ones. (We sum the second-generation populations, since the E stars are a minority.) We computed for each cluster the residuals between the individual $V$ values and $V_{\text {bump }}$, to eliminate the dependence on metallicity and cluster age. We produced histograms both for the whole sample and for the $\mathrm{P}$ and I/E stars separately. The upper panel of Fig. 4 shows the resulting luminosity functions. Since we have only the stars here for which we obtained a spectrum and not the entire RGB, the part below the bump is strongly incomplete, and we do not see the usual increase going towards fainter stars found in photometric works on the RGB luminosity function. However, the bump is 

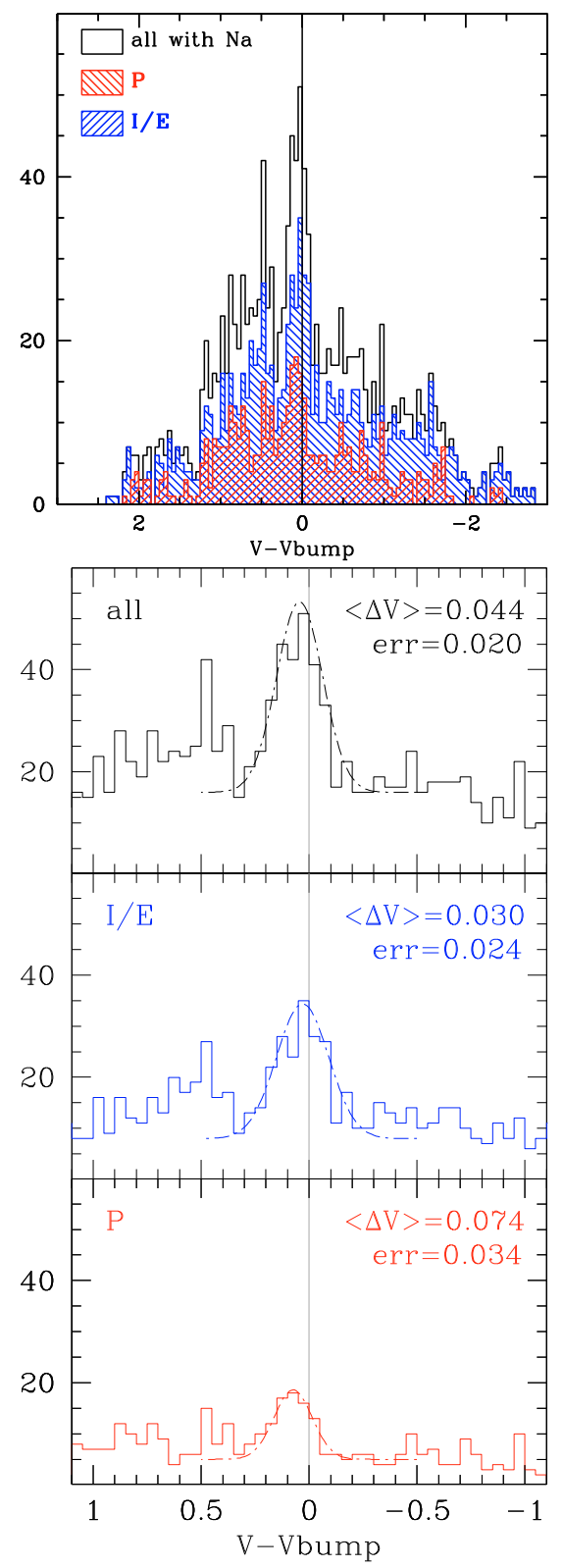

Fig. 4. Upper panel: histogram of the difference in magnitude between individual $V$ and $V_{\text {bump }}$ (for each cluster) for all stars with Na measured (open histogram) and for first generation (red histogram) or second generation (blue histogram). Lower panels: zoom near the RGB bump for all, second-generation (I and E), and first-generation stars, with Gaussians fitting each bump. In each panel the peak of the bump and the associated error are indicated.

visible both in the whole sample and in the two subsamples that show slightly different peak values. The small offset from zero in the histograms is due to the different photometries adopted in the definition of $V_{\text {bump }}$ and in our programme (see below).

To help appreciate this, we present a zoom in the bump region in the lower panels of Fig. 4, separating the three components. Fitting a Gaussian to the bumps we derive a difference in magnitude between P and I, E stars of $0.044 \mathrm{mag}$, in the direction expected from theory, with I, E stars presenting a brighter RGB bump than P stars. Unfortunately, the significance is low, because of the errors on the position of the peaks. A first source of error is the statistics: the bump peak (i.e., above the background) is produced by only about 150 stars, with two thirds in the I, E population and one third in the $\mathrm{P}$ one. To halve the errors in the position of the two separate peaks we should increase the number of stars by at least 3 times, but this is not an immediate possibility because of the large amount of telescope time and analysis implied. Other sources of errors come from the photometry: i) $V_{\text {bump }}$ is measured at best with an uncertainty of 0.05 mag, ii) the magnitudes we use have a small attached error, and iii) we did use a different photometry from the one on which $V_{\text {bump }}$ was determined (although the possible offsets should be amply accommodated within the uncertainty on $V_{\text {bump }}$ ). However, even if we tried to re-determine the bump positions in our photometric data, the situation would not become significantly better. We note in fact that the bump has an intrinsic width of the order of 0.2 mag (Salaris et al. 2002) and that we have already reached that level: the $F W H M$ of the Gaussian used to fit the P population (the one with a single $Y$ value) is $0.21 \mathrm{mag}$ (while the $F W H M$ of the second-generation stars, with varying $Y$ content, is larger, about $0.28 \mathrm{mag}$ ).

To estimate the average difference in the initial He content between the $\mathrm{P}$ stellar population and the $\mathrm{I} / \mathrm{E}$ one, from the measured RGB bump brightness difference, we used the extended set of low-mass stellar models for various assumptions about the initial He content described in Sect. 2.

As a first step, it was assumed that the $\mathrm{P}$ and $\mathrm{I} / \mathrm{E}$ stellar populations have exactly the same heavy elements distribution (case $a$ ). Then for a fixed age of $\sim 12.5 \mathrm{Gyr}$ and iron content $[\mathrm{Fe} / \mathrm{H}]=-1.31$, we computed the synthetic $\mathrm{LF}^{10}$ for the $\mathrm{P}$ population by using a canonical He abundance $(Y=0.248)$, whereas for the I/E population various synthetic LFs were computed by using various He-enhanced abundances in the range from 0.26 to 0.40 . By using these theoretical LFs, we estimated the brightness difference between the RGB bump of the P population and that of the $\mathrm{I} / \mathrm{E}$ one as a function of the difference in the initial $\mathrm{He}$ contents of the two populations. The results of this analysis are shown in Fig. 5. The comparison between the model predictions and the estimated empirical difference suggests a difference in the mean $\mathrm{He}$ content between the $\mathrm{P}$ and the $\mathrm{I} / \mathrm{E}$ sub-populations of about $0.01 \pm 0.01$.

Case $b$ should represent a more significant comparison between theory and observations, because the adopted heavy elements distribution corresponds to a mixture where the sum $(\mathrm{C}+\mathrm{N}+\mathrm{O})$ is enhanced by about a factor of 2 with respect to the reference, $\alpha$-enhanced mixture. This value is consistent although it represents an upper limit - with the results of the spectroscopical analysis performed by Carretta et al. (2005) for the extreme values of the chemical anti-correlations observed in GCs. The RGB bump brightness difference between the P subpopulation and the $\mathrm{I} / \mathrm{E}$ one, when a $\mathrm{CNONa}$ peculiar mixture is assumed for the latter, is shown in Fig. 6 as a function of the He abundance difference. This figure deserves some comments. The RGB bump brightness of the I/E sub-population is fainter than that of the $\mathrm{P}$ sub-population for an He content difference lower than about 0.045 . This occurs because when comparing $\alpha$-enhanced stellar models with those accounting for a CNONa peculiar pattern, we are considering the same iron content $[\mathrm{Fe} / \mathrm{H}]$, and this means that the global metallicity of the stellar models with the peculiar heavy elements distribution is higher than for the reference models. Therefore, as discussed by Pietrinferni et al. (2009), the RGB bump - at fixed age and $[\mathrm{Fe} / \mathrm{H}]$ value - is expected to be fainter in those subpopulations characterised by a peculiar chemical pattern (see

10 To consider the observational errors that can affect the photometric data for each GC in our sample, when computing the synthetic LFs we have considered a photometric error of $\sim 0.02 \mathrm{mag}$. 


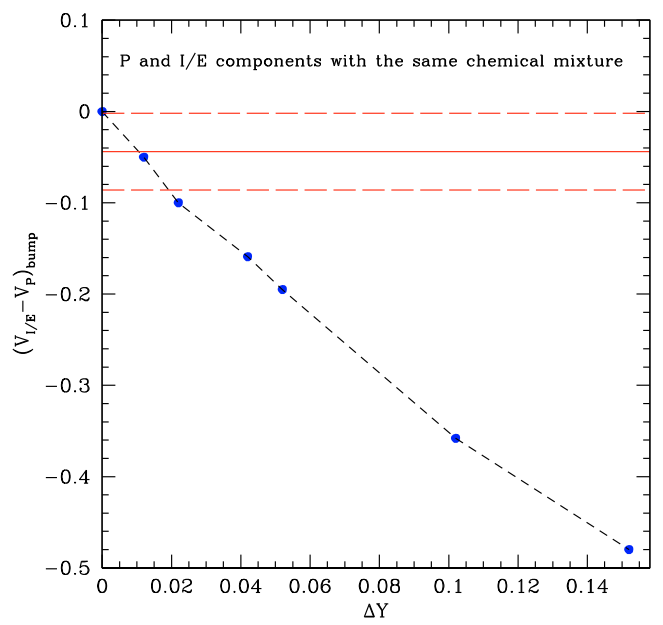

Fig. 5. The $V$-band RGB bump brightness difference between the $\mathrm{P}$ sub-population and the $\mathrm{I} / \mathrm{E}$ one as a function of the He abundance difference between the two sub-populations. In this case, for both subpopulations it has been assumed the same normal, $\alpha$-enhanced heavy elements distribution (case $a$ in Sect. 2). The solid line represents the measured empirical RGB bump luminosity difference between the $\mathrm{P}$ and $\mathrm{I} / \mathrm{E}$ sub-populations, while the long-dashed lines shown the same value at $\pm 1 \sigma$ level.

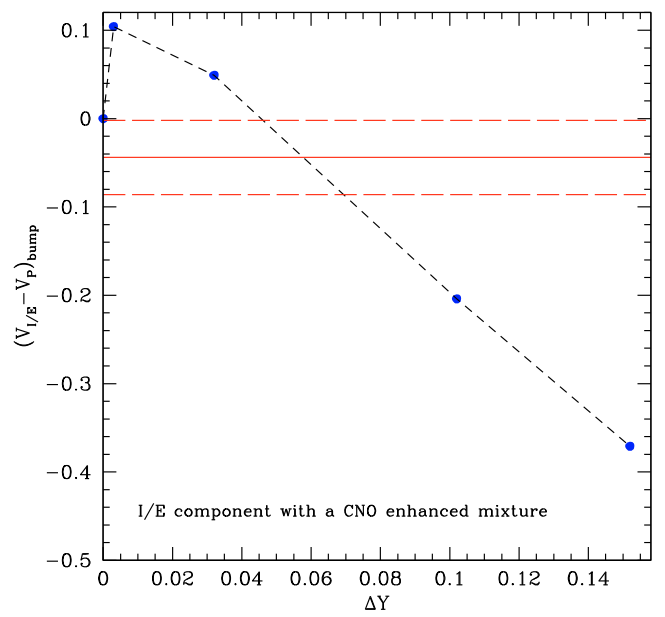

Fig. 6. As in Fig. 5, but taking into account that the $\mathrm{I} / \mathrm{E}$ sub-population is characterised by a peculiar chemical pattern with a CNONa anticorrelation (case $b$ ).

also Salaris et al. 2006). However, for the increasing difference in the He content, the effect of the He abundance on the RGB bump brightness overcomes the one associated to the higher metallicity, and the bump in the I/E sub-population becomes brighter with respect the $\mathrm{P}$ sub-population. Theoretical models shown in Fig. 6 suggest an average difference between the P and the I/E sub-population of $\sim 0.06 \pm 0.015$. This agrees more closely with the estimates obtained in previous sections from independent indicators, even if lower. We consider, however, that NGC 2808 is not in the sample and that this cluster brings a strong signature of $Y$ enhancement.

As stated above, all the stellar models used in this analysis were computed by assuming a constant $[\mathrm{Fe} / \mathrm{H}]$ value regardless of the adopted initial He content. It is in fact expected that both candidate polluters (AGB and FRMS) do not alter the initial iron content. However, it could be worthwhile to check to what extent - if any - the present results are affected by this assumption. For this purpose, we computed suitable stellar models for selected initial He contents, keeping the iron content fixed.
We found that the magnitude difference between the RGB bump of the $\mathrm{I} / \mathrm{E}$ sub-population and that of the $\mathrm{P}$ one is increased by a negligible $0.01 \mathrm{mag}$ for an $\mathrm{He}$ difference between the two sub-populations equal to $\Delta Y=0.05$ and about 0.09 mag for $\Delta Y=0.10$. From Figs. 5 and 6 , it is clear that this change does not affect our previous estimates of the He content difference.

Finally, we wish to draw attention to a possible test: clusters with larger $\Delta Y$ should also display broader RGB bumps. From Figs. 5 and 6 we estimate that a $\Delta Y=0.1$ should correspond to a visible broadening of the bump (since first and second-generation stars should display differences of about $0.20-0.35 \mathrm{mag}$ between their respective bumps). The precise photometry of all their RGB stars should be used. An ideal pair for this comparison are the two GCs NGC 2808 and NGC 6121, which have similar metallicity but very different extensions of the Na-O anticorrelation (Carretta et al., Paper VII) and very different implied $\Delta Y$ (Gratton et al. 2010).

\section{Discussion and summary}

Recently, spreads and splits in GC RGBs have been presented other than the ones (in $V-K$, metallicity and temperature) considered here. They most probably stem from the same phenomenon we are seeing here, since the He-enhanced stars should also show other chemical signatures. For instance, the correlated N-enhancement should be responsible for the effects seen in the colour-magnitude diagrams involving the Johnson $U$ or Strömgren $u$ filters (Marino et al. 2008; Yong et al. 2008). On the other hand, the effect seen in $h k$ photometry, attributed to a spread in calcium by Lee et al. (2009) and demonstrated not to be so by Carretta et al. (2010), still awaits a definite explanation.

We have used information on RGB stars in GCs to infer the plausible He differences implied by the existence of two generations of stars. This is a new approach, since the different methods used in the past to deduce the He content of GCs generally involve the $\mathrm{HB}$, which is the evolutionary phase more sensitive to even small He variations (the two exceptions being $\omega$ Cen and NGC 2808 with their multiple MSs).

Apart from the information coming from photometric data, He can be deduced from the spectra of HB stars, where He lines are visible for temperatures hotter than about $8500 \mathrm{~K}$. Villanova et al. (2009) studied seven HB stars in NGC 6752, using UVES spectra at high resolution and very high S/N. For the four stars where they were able to measure the He line, they obtained a value similar to the cosmological one $(Y=0.245)$. However, these four are all O-rich, Na-poor, while Villanova et al. could not measure He for the only Na-rich, O-poor star, the one that should also be He-enhanced, so the case is not yet settled. However, the photospheric abundances of HB stars hotter than about $11500 \mathrm{~K}$ are altered by atomic diffusion processes and cannot be used directly to measure the original values. For instance, Behr (2003) finds a depletion in moderately hot HB stars in several GCs. In a series of papers (e.g., Moehler et al. 2007), Moehler et al. concentrated instead on the hottest part of the HB (and blue-hook stars in the two GCs - $\omega$ Cen and NGC 2808 - where they are present) and found He-enhancement. This can be attributed either to pollution from a previous generation or to He flash-induced mixing occurring in hot He-flashers, i.e. those stars experiencing the He flash not at the RGB tip but along the white dwarf cooling sequence (see Castellani \& Castellani 1993). A further discussion of this problem in $\omega$ Cen can be found in Cassisi et al. (2009).

In a companion paper (Gratton et al. 2010), we studied the effect of (even a small) difference in He content on the 
morphology of HBs, and found that He is most probably the third parameter governing the $\mathrm{HB}$, after metallicity and age. We refer the reader to that paper for a long and detailed discussion of the effects of He variations, of methods to measure He content (e.g., the R-method: Iben 1968), and of comparison between results obtained through different approaches. In the present paper we limited our analysis to the differences in He that can be deduced from RGB stars.

May we use the present analysis, combined with the previous work on the $\mathrm{Na}-\mathrm{O}$ and other (anti)correlations to try to distinguish between AGB and FRMS as the polluters of the second-generation stars? We need to consider that He is essentially produced in MS for both classes of polluters, while there is a difference for the other "peculiar" elements $(\mathrm{O}, \mathrm{Na}, \mathrm{Mg}, \mathrm{Al}$, $\mathrm{Si}$ ), produced during hot bottom burning or in MS, respectively. However, we still lack important details of stellar evolution (e.g., how much He is dredged up in AGB stars or the dependence on metallicity - via rotation - of FRMS), the numbers are still small, and the inferences are not conclusive. We need to further investigate the dependencies of variations in $\mathrm{He}$ and those other elements on the stars and cluster properties.

In summary, with the present work we have seen that

i) it is possible to also deduce variations of He from RGB stars in GCs when using large samples treated homogeneously;

ii) these variations are generally measurable from colour (here $V-K)$ and $[\mathrm{Fe} / \mathrm{H}]$ only between first-generation $(\mathrm{P})$ and extreme second-generations (E) stars;

iii) the variations implied for the average of the 19 GCs considered range between 0.05-0.10 $(\Delta Y)$; however, the heavyelements mixture assumed for the second-generation stars has to be taken into account (and we have presented two extreme cases);

iv) NGC 2808 is a notable exception because, in this cluster, differences in colour and metallicity are also seen between $\mathrm{P}$ and the intermediate (I) component of second-generation stars. This is perfectly in line with NGC 2808 presenting clear evidence of three He levels also in the MS. For NGC 2808 the deduced $\Delta Y$ values are higher (see Table 3) than for the average of all GCs;

v) similar results can also be obtained when considering the luminosity of the RGB bump. We could only test this method for 14 GCs; unluckily, NGC 2808 is not among them. Within the limits of the rather poor statistics, we found that the implied $\Delta Y$ values are in reasonable agreement with those found from colour and metallicity;

vi) the absolute calibration of the $\Delta Y$ in GCs still needs to be defined.

Acknowledgements. This work was partially funded by the grant INAF 2005 "Experimenting nucleosynthesis in clean environments" and the PRIN MIUR 2007 "Multiple stellar populations in Globular Clusters". S.L. is grateful to the DFG cluster of excellence "Origin and Structure of the Universe" for support. This research has made use of the SIMBAD database, operated at the CDS, Strasbourg, France, and of NASA's Astrophysical Data System. We thank the referee for her/his comments, useful for improving the presentation.

\section{References}

Alonso, A., Arribas, S., \& Martinez-Roger, C. 1999, A\&AS, 140, 261

Alves, D. R., \& Sarajedini, A. 1999, ApJ, 511, 225

Anderson, J., Piotto, G., King, I. R., Bedin, L. R., \& Guhathakurta, P. 2009, ApJ, 697 L58
Bastian, N., \& de Mink, S. 2009, MNRAS, 398, L11

Bedin, L. R., Piotto, G., Anderson, J., et al. 2004, ApJ, 605, L125

Carretta, E. 2006, AJ, 131, 1766

Carretta, E., Gratton R. G., Lucatello, S., Bragaglia, A., \& Bonifacio, P. 2005, A\&A, 433, 597

Carretta, E., Bragaglia, A., Gratton R. G., et al. 2006, A\&A, 450, 523 (Paper I)

Carretta, E., Bragaglia, A., Gratton R. G., Lucatello, S., \& Momany, Y. 2007a, A\&A, 464, 927 (Paper II)

Carretta, E., Bragaglia, A., Gratton, R. G., et al. 2007b, A\&A, 464, 967 (Paper VI)

Carretta, E., Bragaglia, A., Gratton, R. G., et al. 2007c, A\&A, 464, 939 (Paper IV)

Carretta, E., Recio-Blanco, A., Gratton, R. G., Piotto, G., \& Bragaglia, A. 2007d, ApJ, 671, L125

Carretta, E., Bragaglia, A., Gratton, R. G., et al. 2009a, A\&A, 505, 117 (Paper VII)

Carretta, E., Bragaglia, A., Gratton, R. G., \& Lucatello, S. 2009b, A\&A, 505, 139 (Paper VIII)

Carretta, E., Bragaglia, A., Gratton, R., D’Orazi, V., \& Lucatello, S. 2009c, A\&A, 508, 695

Carretta, E., Bragaglia, A., Gratton, R. G., et al. 2010, ApJ, 712, L21

Cassisi, S., Salaris, M., Anderson, J., et al. 2009, ApJ, 702, 1530

Castellani, M., \& Castellani, V. 1993, ApJ, 407, 649

Castellani, M., Castellani, V., \& Cassisi, S. 2005, A\&A, 437, 1017

Catelan, M. 2009, IAU Symp., 258, 209

Cohen, J. G., Persson, S. E., \& Frogel, J. A. 1978, ApJ, 222, 165

D'Antona, F., \& Ventura, P. 2007, MNRAS, 379, 1431

D'Antona, F., \& Caloi, V. 2008, MNRAS, 390, 693

D’Antona, F., Caloi, V., Montalbán, J., Ventura, P., \& Gratton, R. 2002, A\&A, 395, 69

D’Antona, F., Bellazzini, M., Caloi, V., et al. 2005, ApJ, 631, 868

Decressin, T., Meynet, G., Charbonnel C., Prantzos, N., \& Ekstrom, S. 2007, A\&A, 464, 1029

Ferraro, F. R., Messineo, M., Fusi Pecci, F., et al. 1999, AJ, 118, 1738

Glatt, K., Grebel, E. K., Sabbi, E., et al. 2008, AJ, 136, 1703

Gratton, R. G., Lucatello, S., Bragaglia, A., et al. 2006, A\&A, 455, 271

(Paper III)

Gratton, R. G., Lucatello, S., Bragaglia, A., et al. 2007, A\&A, 464, 953 (Paper V)

Gratton, R. G., Carretta, E., Bragaglia, A., Lucatello, S., \& D’Orazi, V. 2010, A\&A, 517, A81

Harris, W. E. 1996, AJ, 112, 1487

Iben., I. Jr. 1968, Nature, 220, 143

Kaviraj, S., Sohn, S. T., O’Connell, R. W., et al. 2007, MNRAS, 377, 987

Lee, Y.-W., Joo, S.-J., Han, S.-I., et al. 2005, ApJ, 621, L57

Lee, J.-W., Kang, Y.-W., Lee, J., \& Lee, Y.-W. 2009, Nature, 462, 480

Mackey, A. D., Broby Nielsen, P., Ferguson, A. M. N., \& Richardson, J. C. 2008, ApJ, 681, L17

Marín-Franch, A., Aparicio, A., Piotto, G., et al. 2009, ApJ, 694, 1498

Marino, A., Villanova, S., Piotto, G., et al. 2008, A\&A, 490, 625

Milone, A., et al. 2008, ApJ, 637, 241

Milone, A. P., Bedin, L. R., Piotto, G., \& Anderson, J. 2009, A\&A, 497, 755

Milone, A. P., Piotto, G., King, I. R., et al. 2010, ApJ, 709, 1183

Moehler, S., Dreizler, S., Lanz, T., et al. 2007, A\&A, 475, L5

Moretti, A., Piotto, G., Arcidiacono, C., et al. 2009, A\&A, 493, 539

Norris, J. E. 2004, ApJ, 612, L25

Pietrinferni, A., Cassisi, S., Salaris, M., \& Castelli, F. 2004, ApJ, 612, 168

Pietrinferni, A., Cassisi, S., Salaris, M., \& Castelli, F. 2006, ApJ, 642, 797

Pietrinferni, A., Cassisi, S., Salaris, M., Percival, S., \& Ferguson, J. W. 2009, ApJ, 697, 275

Piotto, G., Villanova, S., Bedin, L. R., et al. 2005, ApJ, 621, 777

Piotto, G., Bedin, L. R., Anderson, J., et al. 2007, ApJ, 661, L53

Salaris, M., \& Cassisi, S. 2005, Evolution of Stars and Stellar Populations, ISBN 0-470-09220-3. Wiley-VCH

Salaris, M., Cassisi, S., \& Weiss, A. 2002, PASP, 114, 375

Salaris, M., Weiss, A., Ferguson, J. W., \& Fusilier, D. J. 2006, ApJ, 645, 1131

Skrutskie, M. F., Cutri, R. M., Stiening, R., et al. 2006, AJ, 131, 1163

Ventura, P., \& D'Antona, F. 2009, A\&A, 499, 835

Ventura, P., D’Antona, F., Mazzitelli, I., \& Gratton, R. 2001, ApJ, 550, L65

Villanova, S., Piotto, G., \& Gratton, R. G. 2009, A\&A, 499, 755

von Braun, K., \& Mateo, M. 2001, AJ, 121, 1522

Yong, D., Grundahl, F., Johnson, J. A., \& Asplund, M. 2008, ApJ, 684, 1159

Zoccali, M., Cassisi, S., Piotto, G., Bono, G., \& Salaris, M. 1999, ApJ, 518, L49 